\title{
Soil moisture controls on patterns of grass green-up in Inner Mongolia: an index based approach
}

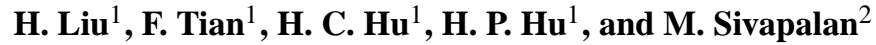 \\ ${ }^{1}$ State Key Laboratory of Hydroscience and Engineering \& Department of Hydraulic Engineering, Tsinghua University, \\ Beijing, 100084, China \\ ${ }^{2}$ Department of Civil and Environmental Engineering, University of Illinois at Urbana Champaign, Urbana, IL 61801, USA \\ Correspondence to: F. Tian (tianfq@tsinghua.edu.cn)
}

Received: 5 October 2012 - Published in Hydrol. Earth Syst. Sci. Discuss.: 12 October 2012

Revised: 1 February 2013 - Accepted: 4 February 2013 - Published: 26 February 2013

\begin{abstract}
Water availability is one of the most important environmental controls on vegetation phenology, especially in semi-arid regions. It is often represented in terms of soil moisture in small-scale studies, whereas it tends to be represented by precipitation in large-scale (e.g., regional) studies. Clearly, soil moisture is the more appropriate indicator for root water uptake and vegetation growth/phenology. Its potential advantage and applicability needs to be demonstrated at regional scales. The paper presents a data-based regional study of the effectiveness of novel water and temperature-based indices to predict spring vegetation greenup dates based on the Normalized Difference Vegetation Index (NDVI) observations in the grasslands of Inner Mongolia, China. The macro-scale hydrological model, VIC (Variable Infiltration Capacity), is employed to generate a soil moisture database across the region. In addition to a standard index based on temperature, two potential hydrologybased indices for prediction of spring onset dates are defined, based on the simulated soil moisture data as well as on observed precipitation data. Results indicate that the correspondence between the NDVI-derived green-up onset date and the soil-moisture-derived potential onset date exhibits a significantly better correlation as a function of increasing aridity compared to that based on precipitation. In this way the soil-moisture-based index is demonstrated to be superior to the precipitation-based index in terms of capturing grassland spring phenology. The results also showed that both of the hydrological (water-based) indices were superior to the thermal (temperature-based) index in determining the patterns of grass green-up in the Inner Mongolia region, indicating water availability to be the dominant control on average. The
\end{abstract}

understanding about the relative controls on grassland phenology and the effectiveness of alternative indices to capture these controls are important for future studies of vegetation phenology change under climate change.

\section{Introduction}

Changes in vegetation phenology and their impacts on ecosystem functioning and agricultural productivity are becoming serious concerns worldwide, especially in the context of global climate change. Phenology is a general biological term that describes recurring natural events, which may include birds migrating back and forth, flowers blooming, and leaves of trees changing color and falling off in autumn. Specifically, in the case of vegetation, phenology includes bud burst, green-up, flowering, plant maturing and finally senescence (which are the various phenophases of vegetation dynamics). Vegetation phenology plays a fundamental role in the functioning of many forest or grassland ecosystems, i.e., variation in phenological stage switch from vegetative growth to reproductive phase could influence the biomass production of the ecosystem (Shinoda et al., 2007). It impacts the hydrological cycle in significant ways by affecting interception and transpiration processes and thus streamflow variability. Their impacts may be detectable in seasonal streamflow variability, especially when precipitation is seasonally uniform. For example, Czikowsky and Fitzjarrald (2004) studied seasonal runoff variability in the northeastern USA, and showed that the signature of phenology was so strong that the phenological changes could be inferred 
directly from flow observations. Thompson et al. (2011) and Ye et al. (2012) showed that not accounting for vegetation phenology can lead to poor predictions of both seasonal evaporation and runoff variability in the northeastern USA, and that a temperature correction that mimics the phenology can help to improve the predictions. More globally, Cayan et al. (2001) showed that in areas of the world with significant deciduous forests, changes in transpiration associated with phenology can contribute to significant changes in seasonal runoff.

Vegetation phenology is influenced by variations in several environmental factors such as temperature, water availability, solar irradiance, and atmospheric oxygen (Lambers et al., 2008), among which water and temperature (collectively called hydrothermal conditions) are the most important ones and are frequently chosen as variables in phenological studies. Studies show that timings of most phenological events in the temperate ecosystems are significantly related to temperature, which means that such events are likely to occur earlier as the climate warms (Sparks et al., 2000). But in semi-arid and arid regions, spring green-up may be limited by either water or temperature availability, and the condition that limits green-up in any specific year may switch between the two. The water condition is likely to limit green-up in dry or warm years, while the thermal condition is likely to limit green-up in cooler or wetter years.

Numerous research efforts have been devoted to quantifying the relationships between phenophases and the controlling or limiting environmental factors. One classic example is the degree day concept, which is defined as the daily accumulation of air temperature above a certain (base) threshold value during a specific pre-seasonal period (see the review by Wang, 1960). This concept attempts to capture the thermal condition needed to be satisfied for vegetation phenological development. It was later extended to include the stress degree day concept to include water stress effects (Idso et al., 1978). Considerable work has been conducted in different geographical and climatic regions to understand and quantify the relationships among climate, soil moisture, and phenology for various types of vegetation, such as tomatoes (Weaver et al., 1988), woody vegetation cover (Seghieri et al., 2009), semi-deciduous (Do et al., 2005), and drought deciduous (Jolly and Running, 2004) vegetation types. Most of these studies have been primarily based on ground measurement of phenological features and environmental variables.

Such ground measurements cannot be easily extrapolated to characterize large-scale regional vegetation phenology, such as regional patterns and broad-scale changes in time. Remote sensing imagery has been widely used over the past two decades for monitoring vegetation dynamics at regional to global scales in view of its synoptic coverage and repeated temporal sampling (Reed et al., 1994; Zhang et al., 2003; Julien and Sobrino, 2009; Yang et al., 2012; among many others). This approach also facilitates other kinds of studies that seek to explore the driving factors behind phenology change in the context of climate change (Piao et al., 2006; Wang et al., 2011; Shen et al., 2011). In these studies, hydrothermal conditions are almost always represented by the degree day concept applied to air temperature (for thermal condition) and precipitation (for water condition). Although soil moisture is a more direct and straightforward index that governs vegetation growth and is frequently used in local studies involving ground measurements, precipitation replaces it in remote sensing-based studies.

Recent advances in hydrological modeling capability, especially at large-catchment or regional scales now provide a sound basis for estimating or predicting large-scale soil moisture with which to analyze and interpret observed space-time patterns in vegetation phenology. A suite of macro-scale hydrological models has been developed that can incorporate sufficient physics to account for the water and energy balances across the land surface. These models can run at spatial and temporal scales much finer than those of climate models and observed meteorological data. The macro-scale hydrological models can already perform reliable simulations of real-time soil moisture and streamflow at daily or sub-daily time steps. Inspired by these recent advances in modeling capability, one of the goals of this research is to demonstrate the advantage of model-produced soil moisture over observed precipitation for analyzing vegetation phenological change.

In this paper we focus on grass phenology. In the case of grasslands, the phenophases include green-up in spring, maturity in summer, senescence in autumn and hibernation in winter. Green-up is the date of onset of photosynthetic activity, the first phenological event, which plays an important role in the whole life cycle of grasses, and can influence the biomass productivity, biodiversity evolution, water balance and carbon balance at local, regional and global scales. Here we focus on the spring grass green-up onset, which is likely to be affected by variations in thermal and moisture supply. The aim is to investigate the soil moisture and temperature controls on grass green-up onset, including the feasibility of using a soil-moisture-based index for its analysis and its advantages over a precipitation-based index. The Inner Mongolia grassland area (IMGA) is selected as the study area. The Advanced Very High Resolution Radiometer (AVHRR) Normalized Difference Vegetation Index (NDVI) data provided by the Global Inventory Monitoring and Modeling Studies (GIMMS) are used to characterize grass phenology. The distributed Variable Infiltration Capacity (VIC) hydrological model is applied over the IMGA region to generate the distributed soil moisture data used to construct the soilmoisture-based index.

This paper is organized as follows: in Sect. 2 we introduce the study area and the data sources, the method used for detection of grass green-up from NDVI data, and outline the basics of the VIC model used for generating soil moisture fields. We propose two different indices to characterize water availability, based on soil moisture and precipitation. In Sect. 3 we present the hydrological and meteorological 
results and additional results that bring out the spatial patterns and temporal trends in the timing of grass green-up in the study area under an apparent warming trend found in the region. We also use the results of the analysis to demonstrate the superiority of the soil-moisture-based index over the precipitation-based index, and together with these investigate if and how the dominant factors that control grass greenup vary in space regionally and in time. This is followed by a brief summary and conclusions in Sect. 4 .

\section{Methodology}

\subsection{Study area and data}

The IMGA is located in northern China (Fig. 1). The area has an average altitude of $1100 \mathrm{~m}$ a.s.l. (above sea level) and an area of $3.1 \times 10^{5} \mathrm{~km}^{2}$. The meteorological data are from the China Meteorological Data Service System (http: //cdc.cma.gov.cn/). The study area has 21 stations (see Fig. 1) with records from 1982 to 2006 . The mean annual temperature ranges from -3 to $7{ }^{\circ} \mathrm{C}$, which increases from north to south. Annual precipitation ranges from 100 to $400 \mathrm{~mm}$, with a mean value of $300 \mathrm{~mm}$, and principally falls between May and September. The mean annual pan evaporation (E20) is $2030 \mathrm{~mm}$. The climate is dry (arid), and the study area belongs to the inland region of China. The dominant vegetation type in the IMGA is grass, as shown in Fig. 1, which is classified according to the MODIS product MOD12C1. The AVHRR NDVI time series data of GIMMS (http://glcf.umiacs.umd.edu/data/gimms/) from 1982 to 2006 are used to capture the timing of grass spring green-up onset. The Sandaohezi hydrological station on Luan River (see Fig. 1) close to the study area is chosen to assess the predictions of the VIC hydrological model.

\subsection{Macro-scale hydrologic model}

The VIC model (Liang et al., 1994) was used in this study to simulate the space-time dynamics of soil moisture across the region. VIC is a physically based, semi-distributed macroscale hydrological model that balances both water and surface energy within a grid cell. The VIC model can account for sub-grid variability of land surface vegetation classes and soil moisture storage capacity, and includes an infiltration algorithm that accounts for the areal extent of soil saturation. Soil layer depths, infiltration, and baseflow parameters have to be calibrated. VIC has been widely used for streamflow simulation and drought monitoring in large river basins globally, and across China (e.g., Yuan et al., 2004; Sheffield and Wood, 2007). In this study the VIC model was implemented across China at a $0.5^{\circ}$ resolution from 1951 to 2010 . The model parameters were calibrated using the method proposed by Xie et al. (2007).

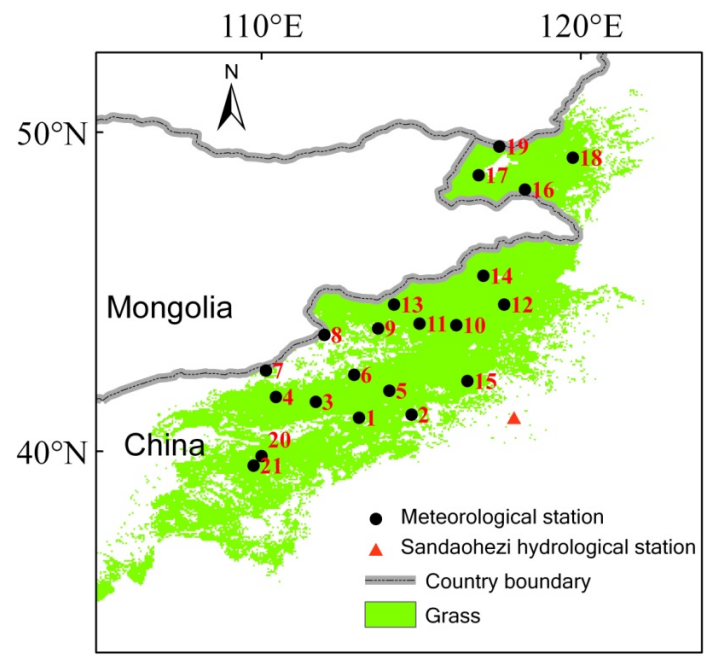

Fig. 1. Study area and gauging stations.

\subsection{Green-up onset date and the Holdridge aridity index}

Zhang et al. (2003) defined the green-up onset date (GUD) as the day when the rate of change of the NDVI curvature (RCC) reaches its first local maximum value (see Fig. 2). We adopted this method to capture the grass GUD from the satellite images. The NDVI data marked as having good values were averaged within a $3 \times 3$ pixel window. A SavitzkyGolay filtering procedure was applied to each annual time series of NDVI to obtain a smoothed series (Chen et al., 2004; Shen et al., 2011). Afterwards, the smoothed series was fitted by the following four-parameter logistic function (Zhang et al., 2003; Shen et al., 2011):

$\operatorname{NDVI}(t)=c /\left(1+e^{a+b t}\right)+d$,

where the symbols $a, b, c$ and $d$ are best-fit model parameters, with $d$ being the initial background NDVI value, and $c+d$ being the maximum NDVI value. An expression for the rate of change of curvature (RCC) was derived from Eq. (1) as follows:

$$
\begin{aligned}
\mathrm{RCC}= & b^{3} c z \times 3 z(1-z)(1+z)^{3}\left[2(1+z)^{3}+b^{2} c^{2} z\right] / \\
& {\left[(1+z)^{4}+(b c z)^{2}\right]^{2.5}-b^{3} c z \times(1+z)^{2} } \\
& \left(1+2 z-5 z^{2}\right) /\left[(1+z)^{4}+(b c z)^{2}\right]^{1.5}
\end{aligned}
$$

where $z=e^{a+b t}$.

It should be noted that for some extremely dry years and/or stations, the GUD could not be determined at all by the RCC method described above. In fact, the grassland might not even green up in the dry years due to insufficient moisture supply. To investigate the possible connection between grass green-up patterns and aridity, we characterized the relative 


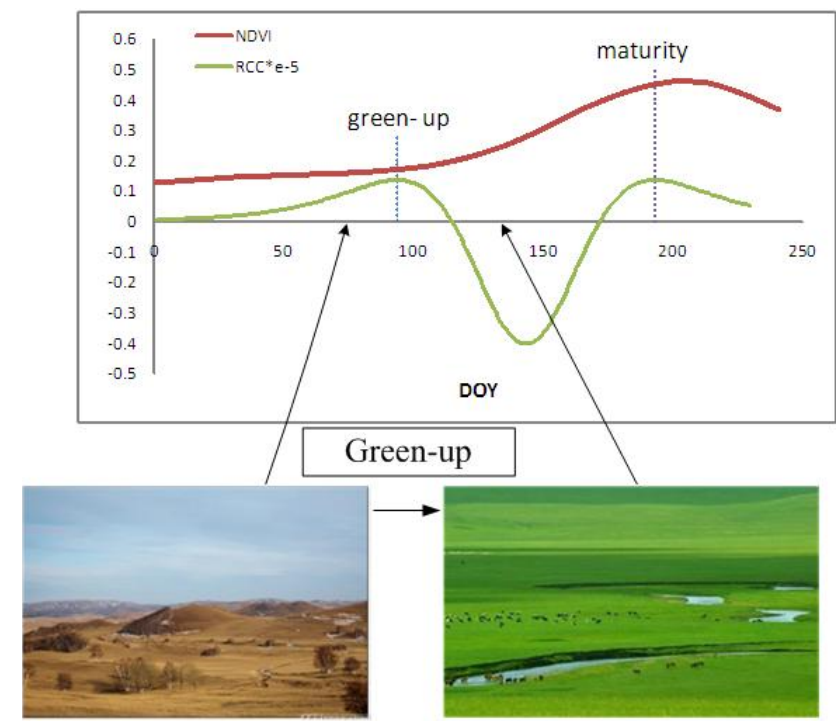

Fig. 2. RCC of fitted NDVI series by a four-parameter logistic function (the two pictures are not exactly taken at the same place, but both are in Inner Mongolia).

aridity of the stations with the Holdridge aridity index (HAI) (Holdridge, 1947). The HAI index is defined as follows:

$\mathrm{HAI}=58.93 \times \sum T / 365 \times P$,

where $P$ is the annual precipitation ( $\mathrm{mm}$ ); $T$ is the daily mean temperature within the range of 0 to $30^{\circ} \mathrm{C}$, which is excluded from the calculation when $T$ is less than the lower limit and assigned a value of $30^{\circ} \mathrm{C}$ when $T$ is greater than the upper limit.

\subsection{Proposed environmental indices}

The factors influencing vegetation green-up include irradiance, temperature, soil moisture etc., amongst which the thermal and water conditions have been of most concern to large-scale vegetation phenology researchers (Yu et al., 2003; Jolly and Running, 2004). However, the relationship between green-up onset and the controlling factors can be complex, and the limiting environmental factor may shift (between places and between years) according to the prevailing hydrothermal conditions. Taking the example of water availability, depending on their biophysical characteristics and physiological features of the vegetation, one can expect that dormant vegetation will not green-up immediately upon seeing the first rainfall - a behavior characteristic that can be explained by way of self-protection to avoid water deficit in the subsequent growth stage, in case the rainfall does not persist. This means that water accumulation through precipitation must reach a threshold over some time span before vegetation would green up (Zhang and Liang, 2007). These notions of accumulation, threshold and time span apply to other environmental factors as well, such as temperature.
In the past, to explore the relationships between the environmental factors and vegetation green-up, it has been common to accumulate the indicator variables over a fixed time span (Yu et al., 2003), for example the (typical spring) month of May. However, this has the drawback that it is quite possible that vegetation green-up may have already occurred by the middle of May. Accumulation over the whole month of May complicates the relationship and contributes to poor predictability. Recently, a more flexible and appropriate time span was developed by Shen et al. (2011) for the concept of thermal spring onset date (TSO). The TSO was defined as the date when the green-up can potentially begin, which means the daily temperature accumulated from a certain day to the TSO is above a threshold required for vegetation greenup. Similarly (and in addition) to the definition of TSO, we propose and compare two alternative indices that account for the control of water availability on the potential GUD, i.e., the date by which the water requirement for the onset of green-up is satisfied. First, we define the soil moisture spring green-up onset date (SMSO) as the date by which the accumulated soil moisture storage reaches a threshold that the grass needs to green-up. Secondly, we also adopt an alternative precipitation-based index, the precipitation spring greenup onset date (PSO), defined as the date by which the accumulated precipitation reaches a depth threshold that grass needs to green up. In each case (temperature, soil moisture, precipitation), there are three components to the definition of the indices: (1) the end day of the accumulation period; (2) the time span; (3) the threshold. In this study we use the GUD detected from the remote sensing data as the end day of the accumulation period. For any chosen time span, we use the mean of the 25-yr accumulation over that time span as the corresponding threshold value. We then optimize the magnitude of the time span through the use of a maximization procedure proposed by Shen et al. (2011). Details of the optimization procedure are described below.

\subsubsection{Accumulation threshold}

If the time span chosen is $N_{\text {days }}$, the corresponding accumulation threshold is defined as the 25-yr (1982 to 2006) average of the $N_{\text {days-accumulated soil moisture (termed as }}$ $\left.\mathrm{ASM}_{N}\right)$ :

$\operatorname{ASM}_{N}=1 / 25 \sum_{\text {year }=1982}^{2006}\left(\sum_{i=\mathrm{GUD}_{\text {year }}-N}^{\mathrm{GUD}_{\text {year }}-1} \mathrm{SM}_{\text {year }}^{i}\right)$,

where $\mathrm{SM}_{\text {year }}^{i}$ is the soil moisture for the $i$-th DOY in a specific year.

Given $N_{\text {days }}$ and $\mathrm{ASM}_{N}$, we then define the SMSO of a certain year (i.e., denoted $\mathrm{SMSO}_{1982}^{N_{\text {days }}}$ for the year 1982) as the last day of an $N_{\text {days }}$ window that fulfills the following conditions: (a) the accumulated daily soil moisture reaches $\mathrm{ASM}_{N}$, and (b) it is the earliest time window that fulfills condition (a). As shown in Fig. 3a, if $N_{\text {days }}$ is 5, we slide a 5-day 
moving window starting from the first day of 1982 until the accumulated soil moisture reaches $\mathrm{ASM}_{5}$, upon which we record the last day of the current window as $\mathrm{SMSO}_{1982}^{5}$.

\subsubsection{Variable time span}

After estimating the SMSO for each year between 1982 and 2006, we have an annual time series of SMSO value for a given $N_{\text {days }}$ :

$\boldsymbol{S M S O} \boldsymbol{O}^{N_{\text {days }}}=\left\{\mathrm{SMSO}_{1982}^{N_{\text {days }}}, \mathrm{SMSO}_{1983}^{N_{\text {days }}}, \ldots, \mathrm{SMSO}_{2006}^{N_{\text {days }}}\right\}$.

We can repeat this procedure for several values of $N_{\text {days }}$, and in this way obtain several time series of SMSO values. We assume that the potential GUD accounting for water availability should be positively cross-correlated to the GUD time series detected from the remote sensing data. Guided by this thinking, the time span of accumulation (the number of days, $N_{\text {days }}$, preceding the known GUD) was then determined as the value of $N_{\text {days }}$ (Note: $1 \leq N_{\text {days }} \leq 90$ in steps of one day) that produces the maximum cross-correlation coefficient between the annual time series of SMSO ${ }^{N_{\text {days }}}$ and GUD. The maximization algorithm can be formally presented as follows:

Maximize : cross-correlation $\left(\boldsymbol{G U} \boldsymbol{D}, \boldsymbol{S M} \boldsymbol{S} \boldsymbol{O}^{N_{\text {days }}}\right)$

$\boldsymbol{G U} \boldsymbol{D}=\left\{\mathrm{GUD}_{1982}, \mathrm{GUD}_{1983}, \ldots, \mathrm{GUD}_{2006}\right\}$.

The GUD included in Eqs. (6) and (7) is a vector consisting of annual GUD values from 1982 to 2006.

Figure $3 b$ presents the maximizing procedure in a schematic form, where the black bold line represents the GUD series from 1982 to 2006, and the other lines stand for $\boldsymbol{S} \boldsymbol{M S} \boldsymbol{S}^{N_{\text {days }}}$ with different $N_{\text {days. }}$. After comparing the correlation between $\boldsymbol{G} \boldsymbol{U} \boldsymbol{D}$ and $\boldsymbol{S} \boldsymbol{M} \boldsymbol{S} \boldsymbol{O}^{N_{\text {days }}}$, we could get the largest correlation coefficient and the corresponding $\boldsymbol{S} \boldsymbol{M S} \boldsymbol{O}^{N_{\text {days }}}$. In Fig. $3 b$ the optimal accumulation time span is 5 days, which is labelled as $\boldsymbol{S} \boldsymbol{M} \boldsymbol{S} \boldsymbol{O}^{5}$ and shown as a red bold line, and the corresponding accumulation threshold is labeled as $\mathrm{ASM}_{5}$.

Following this maximization process, the $\boldsymbol{S M S O}$, $\mathrm{ASM}_{N_{\mathrm{SM}}}$, and corresponding optimal value of $N_{\text {days }}$ (denoted as $N_{\mathrm{SM}}$ ), and the resulting optimal cross-correlation coefficient between the GUD and SMSO time series are determined for each measurement station. The same procedure is also followed for the case of precipitation and temperature, and correspondingly we obtain the values of $\boldsymbol{P S O}$, $\mathrm{AP}_{N_{\mathrm{P}}}, N_{\mathrm{P}}$, and the optimal cross-correlation coefficient between GUD and PSO time series, and likewise for the values of $\boldsymbol{T} \boldsymbol{S} \boldsymbol{O}, \mathrm{AT}_{N_{\mathrm{T}}}, N_{\mathrm{T}}$, and the optimal cross-correlation coefficient between GUD and TSO time series. These will be used later for a comparative assessment of the three indices. (a)
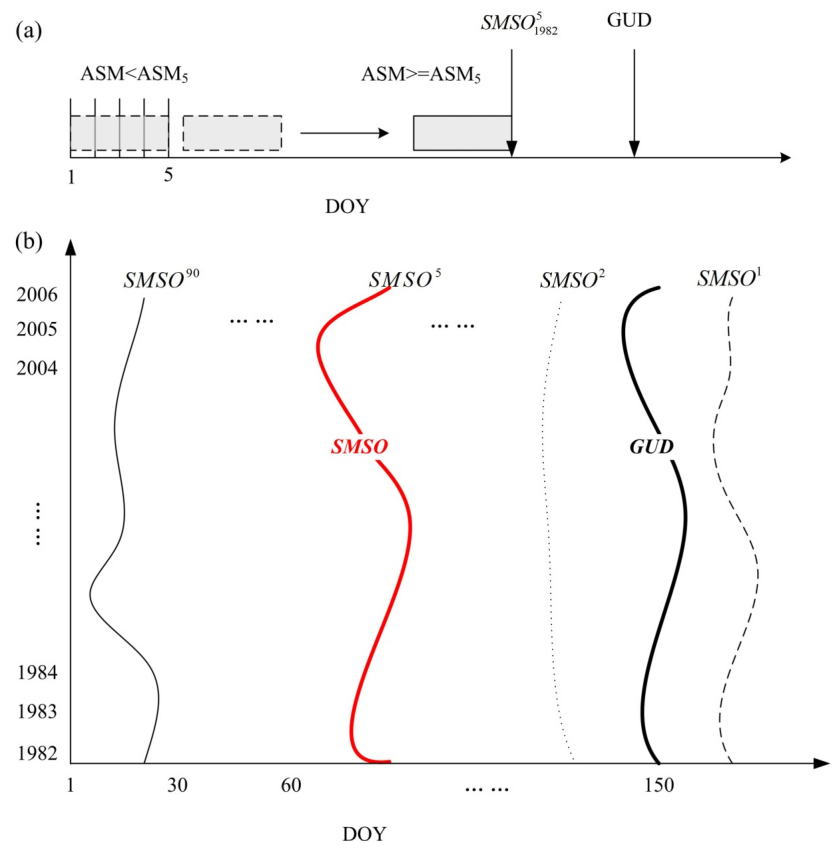

Fig. 3. (a) Schematic chart of the definition of SMSO, a fixed window (e.g., 5-day) moving afterward from the first day of the year (e.g., 1982) until the accumulated soil moisture reaches the threshold $\mathrm{ASM}_{5}$, then the last day of the window is $\mathrm{SMSO}_{1982}^{5}$ (b) The maximizing process to get the optimal length of accumulation period. For each window (e.g., 1 to 90), we get the corresponding time series of SMSO from 1982 to 2006 (i.e., $\mathrm{SMSO}^{1}, \ldots, \mathrm{SMSO}^{90}$ ), after calculating the correlation between each SMSO time series with GUD time series, we could pick out the SMSO time series with the best correlation coefficient, then the corresponding window size is the optimal length of accumulation period (i.e., 5).

\subsection{Regression of GUD based on a dominant thermal/hydrological index}

The estimation procedure described above for the three environmental indices considered water and temperature as alternative controls. In reality their effects on the GUD must be considered together, not in isolation (i.e., one or the other). If they are to be considered together and regressed together against estimated GUD values, then the question arises as to what accumulation time span to use. We address this problem in this study by alternatively choosing water or temperature as the dominant control, and the remaining one (as the case may be) as a secondary control. In each case the chosen time span is that of the dominant control. For example, if soil moisture is assumed as the dominant control, then both soil moisture and air temperature are accumulated over $N_{\text {SM }}$ days ahead of the GUD each year, as defined below:

$\mathrm{SM}_{\text {year }}^{N_{\mathrm{SM}}}=\sum_{i=\mathrm{GUD}-1}^{\mathrm{GUD}-N_{\mathrm{SM}}} \mathrm{SM}_{\text {year }}^{i}$ 


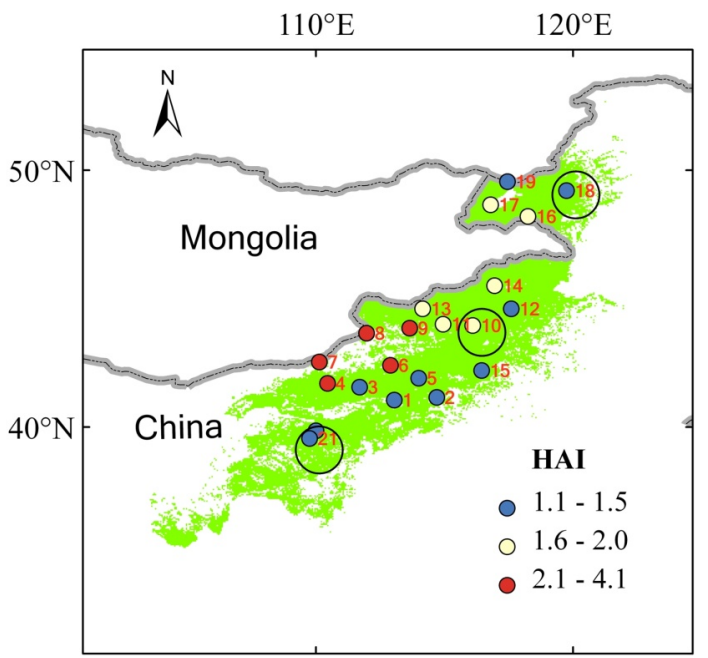

Fig. 4. The Holdridge aridity index of meteorological stations in Inner Mongolia grassland area.

$T_{\text {year }}^{N_{\mathrm{SM}}}=\sum_{i=\text { GUD }-1}^{\text {GUD }-N_{\mathrm{SM}}} T_{\text {year }}^{i}$,

where the year is $\{1982,1983, \ldots, 2006\}$. In this way we obtain the following vectors and regress the GUD values on both $\mathrm{SM}^{N_{\mathrm{SM}}}$ and $T^{N_{\mathrm{SM}}}$ simultaneously through multiple regression:

$\boldsymbol{S} \boldsymbol{M}^{N_{\mathrm{SM}}}=\left\{\mathrm{SM}_{1982}^{N_{\mathrm{SM}}}, \mathrm{SM}_{1983}^{N_{\mathrm{SM}}}, \ldots, \mathrm{SM}_{2006}^{N_{\mathrm{SM}}}\right\}$

$\boldsymbol{T}^{N_{\mathrm{SM}}}=\left\{T_{1982}^{N_{\mathrm{SM}}}, T_{1983}^{N_{\mathrm{SM}}}, \ldots, T_{2006}^{N_{\mathrm{SM}}}\right\}$

$\boldsymbol{G} \boldsymbol{U} \boldsymbol{D}_{\mathrm{reg}}^{\mathrm{SM}}=\beta_{1} \mathrm{SM}^{N_{\mathrm{SM}}}+\beta_{2} T^{N_{\mathrm{SM}}}+\beta_{3}$

$r_{\mathrm{SM}}^{\mathrm{GUD}}=\operatorname{correl}\left(\boldsymbol{G U} \boldsymbol{D}, \boldsymbol{G U} \boldsymbol{D}_{\mathrm{reg}}^{\mathrm{SM}}\right)$,

where $\beta_{1}, \beta_{2}$, and $\beta_{3}$ are the regression coefficients, and $r_{\mathrm{SM}}^{\mathrm{GUD}}$ is the correlation between NDVI-derived GUD and the regressed GUD $\left(\boldsymbol{G} \boldsymbol{U} \boldsymbol{D}_{\mathrm{reg}}^{\mathrm{SM}}\right)$. Note that both soil moisture and precipitation are used as alternative controls to represent water availability. Using a similar procedure (as above), $P^{N_{\mathrm{P}}}$, $T^{N_{\mathrm{P}}}$, and $r_{\mathrm{P}}^{\mathrm{GUD}}$ are calculated assuming precipitation as the dominant control (and temperature as secondary control). We then repeat the analysis and estimate $\boldsymbol{S} \boldsymbol{M}^{N_{\mathrm{T}}}, \boldsymbol{T}^{N_{\mathrm{T}}}$, and $r_{\mathrm{T}}^{\mathrm{GUD}}$ by assuming air temperature as the dominant control and soil moisture as the secondary control. In this way three different regressions are carried out, producing three different correlation coefficients, which are then compared against each other.

\section{Results}

\subsection{Hydrological and meteorological results}

The Nash-Sutcliffe efficiency coefficient (NSCE) of monthly streamflow simulation results at the Sandaohezi station is 0.66. This is deemed sufficient for the purposes of this study, and the soil moisture output of the model is used in constructing the soil moisture index as a surrogate for water availability for the vegetation.

Estimation of the aridity index HAI within the study region indicates that the region is characterized by a dry climate, with HAI values ranging from 1.0 to 5.0 (see Fig. 4 for the location of the gauges as well as the spatial patterns of HAI within the study area). Measurement stations 6 to 9 in the northern middle part of the study area have the highest HAI. These four gauges were therefore excluded from further analysis because it was found that their unusual life cycle could not be detected from remote sensing data using the RCC method.

\subsection{GUD patterns and the shift of dominant controls in Inner Mongolia}

We implemented the GUD detection method (Eqs. 1 and 2) to the remaining 17 gauging stations, and estimated their GUD values. For illustration we have presented the results for three stations that are located at the northern (station 18), middle (station 10) and southern (station 21) parts of the study area, and have different HAI values (their locations being denoted in Fig. 4 by big circles). The resulting annual time series of GUD values for these three stations are presented in Fig. 5 for the years 1982 to 2006. They show that annual GUD values can vary over a wide range -90 to 160 (day of the year, or DOY) - and that there does not appear to be much concurrence between the temporal patterns between the three gauge locations. Whereas Fig. 5 presented illustrative results of GUD variability in time, the GUD values also exhibit considerable spatial (regional) variability. In order to illustrate this, in Fig. 6 we present the spatial pattern of the 25-yr mean GUD values across the region, with the mean GUD estimated from the NDVI data falling in the range of 116 to 137 (DOY) among the 17 gauging stations. Both of these results show that GUD values in this region exhibit considerable spatial (regional) and temporal (inter-annual) variability.

Based on temperature records between 1982 and 2006 the study region has also experienced a warming of the climate. Figure 7a displays a positive (increasing) trend in the temperature in all meteorological stations. Precipitation, however, shows quite variable trends between the stations. Most meteorological stations exhibit a decreasing (negative) trend in precipitation, whereas a few (6 of the 21) in the southwestern part of the area close to the Qinghai-Tibetan Plateau, show an increasing (positive) trend. The corresponding spatial patterns in temporal changes in the GUD values, presented in Fig. $7 \mathrm{~b}$, show prolonged (positive) trends in the middle part of the study region and advanced (negative) trends in the northern as well as southern parts. These patterns of temporal GUD trends are also in agreement with results obtained by Yu et al. (2003). From the above results, one can see that although the temperature in the whole area has been rising 

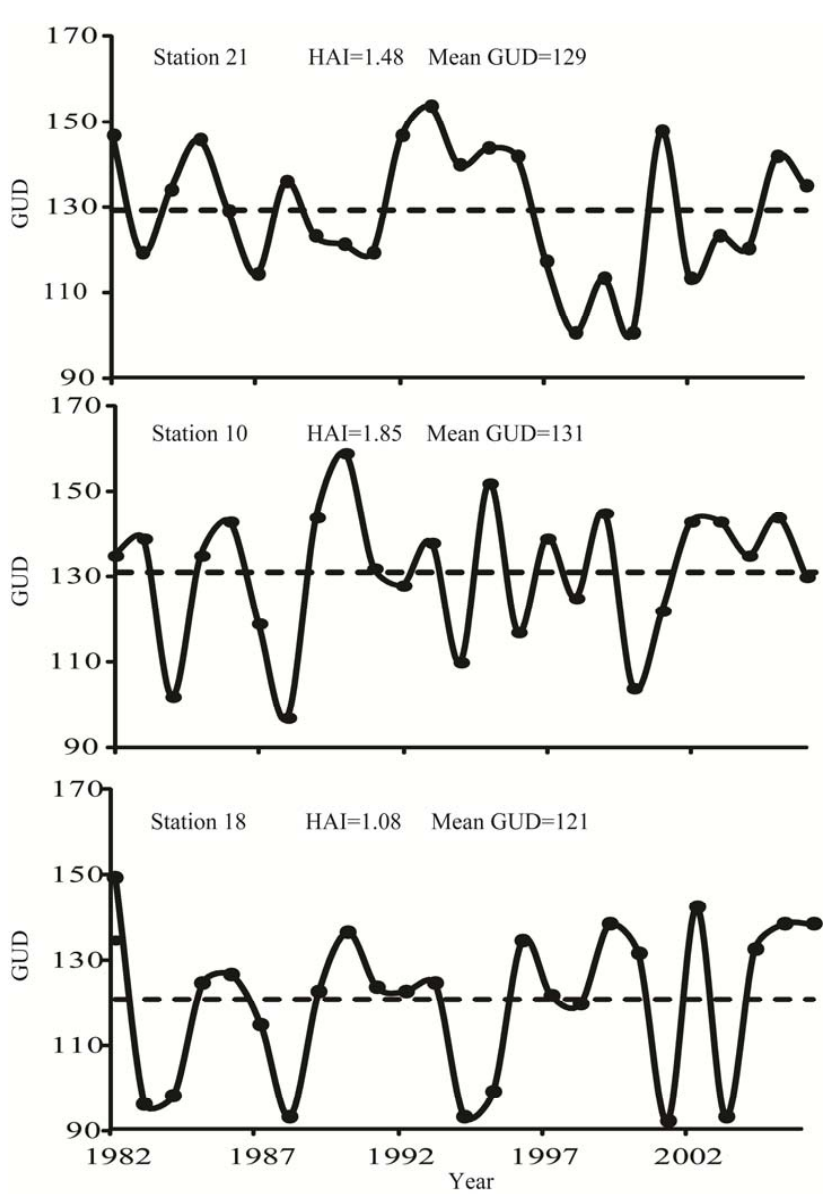

Fig. 5. Temporal pattern of GUD at stations numbered 21, 10, and 18 .

over the past $25 \mathrm{yr}$, the spatial and temporal trends in GUD are highly variable, suggesting that grass green-up in this region may be influenced by factors other than the thermal condition, the most important of which is the water condition.

We illustrate this using data taken from one station within the study region. Figure 9 presents the time series of (smoothed) NDVI, air temperature, (smoothed) precipitation and also modeled soil moisture for station 10 for two consecutive years, 1997 and 1998. The estimated GUDs from the NDVI data were 133 and 128 DOY, respectively, and are marked on the figure as vertical lines. In 1997, as can be seen in Fig. 8a, the soil moisture was already at a high value since March (perhaps due to carry-over from the previous year), but the temperature was too low for green-up until it hit a threshold, so the dominant control in 1997 may have been temperature. In 1998, shown in Fig. 8b, the temperature was uniformly high in April, yet the grass did not green up until the accumulation of soil moisture due to precipitation rose up and remained high consistently, which suggests that the dominant control in 1998 was in fact water (soil moisture). These two examples illustrate the competing controls on GUD, although this kind of evaluation is very subjective and time

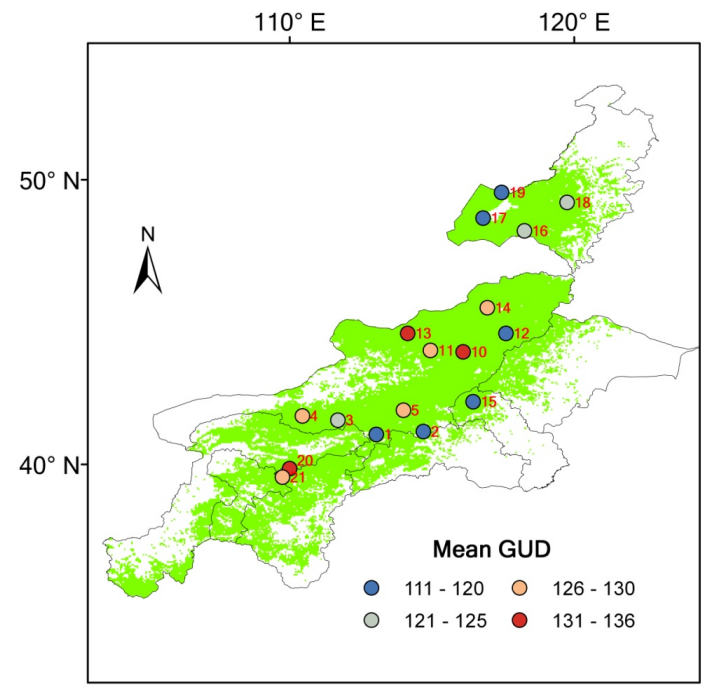

Fig. 6. The spatial pattern of 25-yr averaged GUD of the stations.

consuming. Could there be an alternative way to determine the dominant controls that mimic the same decision-making processes or rules that grasses possibly adopt during their green-up phase? In the next section we assess the power of the environmental indices proposed earlier to capture the underlying controls objectively and make prior predictions.

\subsection{Soil moisture vs. precipitation as the basis for a water index}

The time series of SMSO, PSO and TSO for station $10 \mathrm{ob}-$ tained using the estimation procedure described in Sect. 2.4 are presented in Fig. 9 for illustration, along with the corresponding GUD values estimated from the NDVI data. These results indicate, for station 10 , that the PSO values lie mostly above the GUD values (with very little overlap), whereas the SMSO and TSO values seem to fluctuate above and below the GUD values, with considerable overlap. On the basis of these results, we speculate that in this case the SMSO and TSO indices are more appropriate indices to explain the interannual shifts of GUD, and that the precipitation-based index does not have much explanatory power to account for the effect of water availability in relation to the soil-moisturebased index.

We expect that the correlation between the GUD and the soil-moisture-determined potential green-up onset date (SMSO) would be better correlated in more arid areas, as characterized by the aridity index, HAI. We plotted the correlation coefficients between GUD and SMSO obtained at each of the 17 stations as a function of their HAI. In addition, we also plotted the estimated correlation coefficients between GUD and PSO in the same figure so as to assess which of the two indices produces a superior predictive performance. The results are presented in Fig. 10, and show that the correspondence between the GUD and SMSO as a function of HAI is 

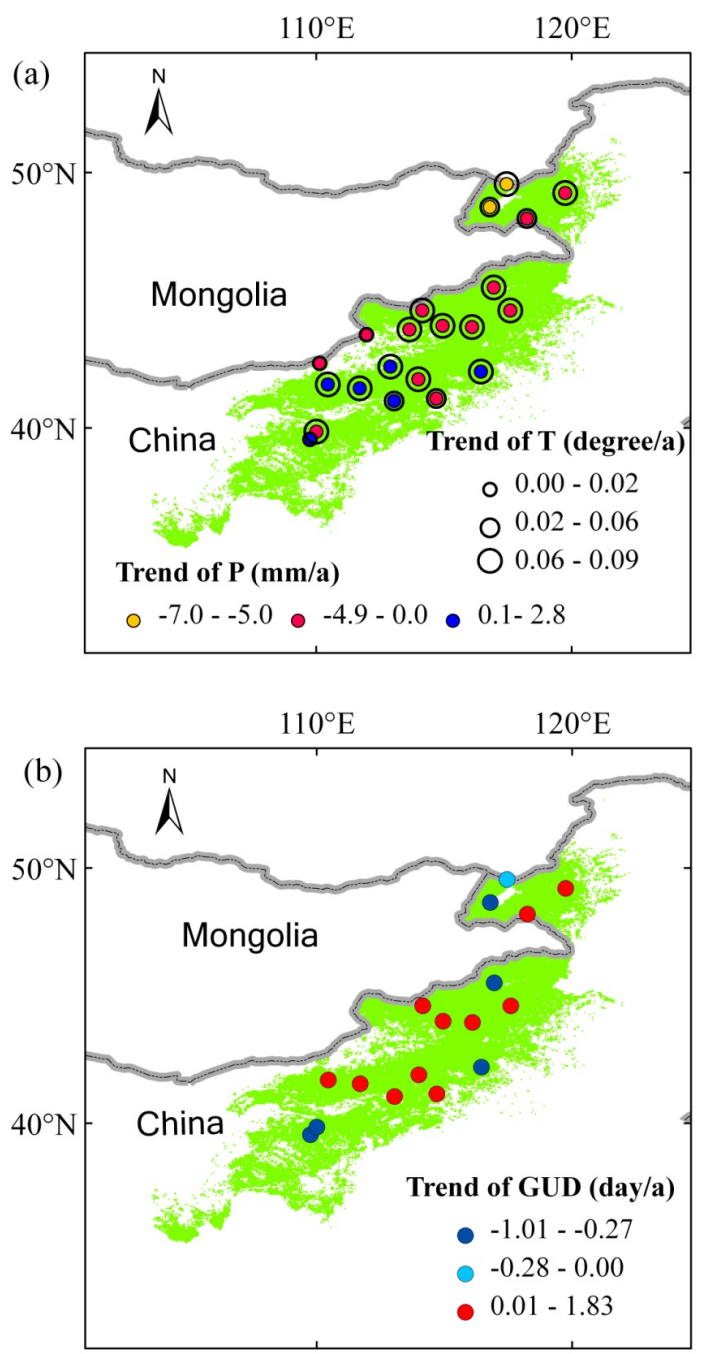

Fig. 7. (a) Trend of air temperature and precipitation in Inner Mongolia from 1982 to 2006; (b) trend of GUD in Inner Mongolia from 1982 to 2006.

stronger, with a coefficient of determination of 0.52 , whereas the correspondence between GUD and PSO as a function of HAI is rather week, with a coefficient of determination of only 0.02 . This result shows that the soil-moisture-based index (SMSO) is superior to the precipitation-based index (PSO) as a measure of water availability for capturing observed GUD variations.

\subsection{Regression results based on dominant thermal/ hydrological indices}

The regression results obtained for the 17 stations using the multiple regression procedure described in Section 2.5 are shown in Fig. 11. The averaged $r_{\mathrm{SM}}^{\mathrm{GUD}}$ and $r_{\mathrm{P}}^{\mathrm{GUD}}$ values over the 17 stations are, respectively, 0.879 and 0.809 , which are higher than the averaged correlation $r_{\mathrm{T}}^{\mathrm{GUD}}(0.791)$ based on temperature dominance. Figure 11 also indicates
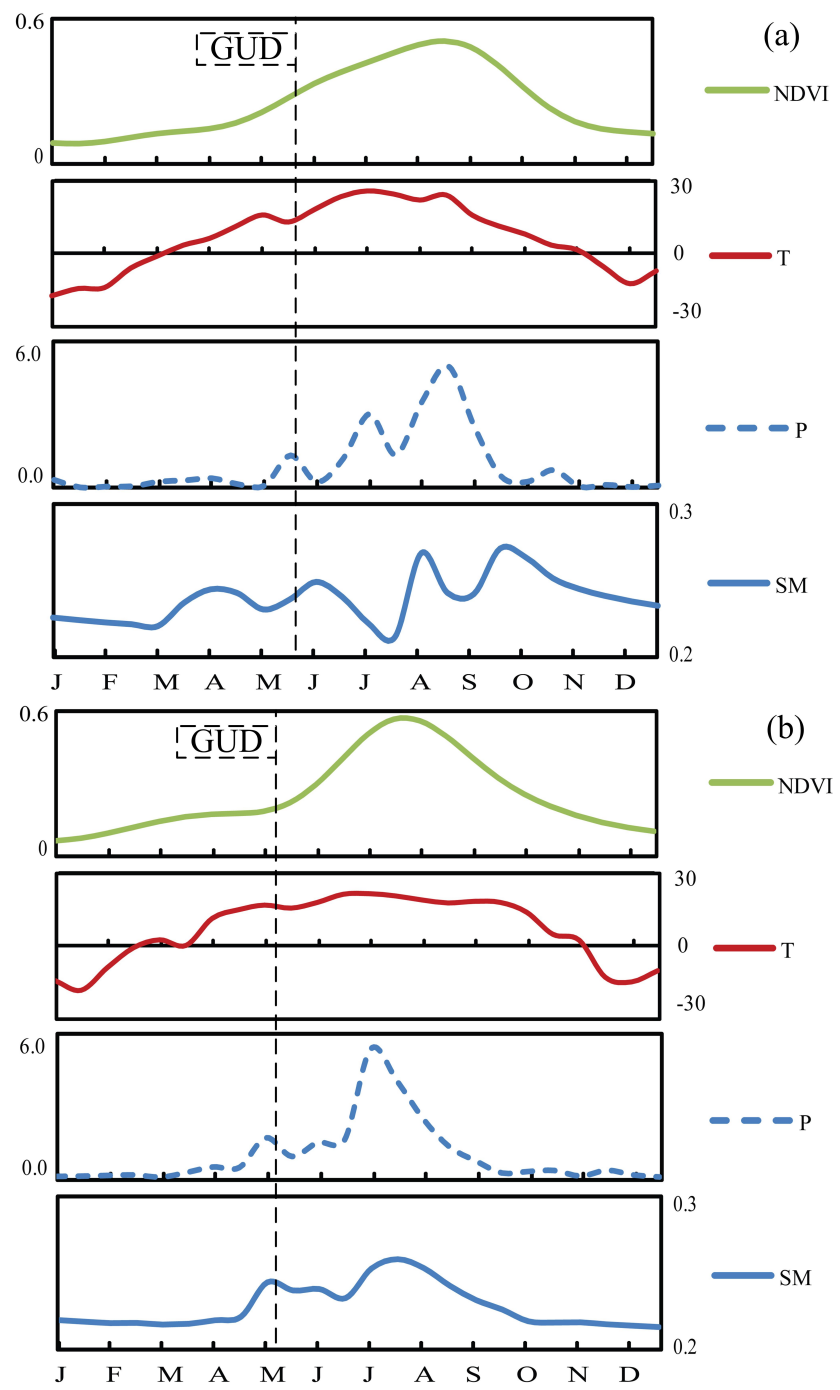

Fig. 8. Annual series of NDVI, simulated soil moisture, precipitation and air temperature of 1997 (a) and 1998 (b) at station number 10 (the captions $\mathrm{J}$ to $\mathrm{D}$ in the $\mathrm{x}$-axis stands for January to December).

persistently higher positions for the hydrological lines (representing $r_{\mathrm{SM}}^{\mathrm{GUD}}$ and $r_{\mathrm{P}}^{\mathrm{GUD}}$ ) than for the thermal line (representing $r_{\mathrm{T}}^{\mathrm{GUD}}$ ), implying that the vegetation life cycle in the IMGA may be dominated more by the water condition than by the thermal condition. Furthermore, a slightly larger value of $r_{\mathrm{SM}}^{\mathrm{GUD}}(0.879)$ than $r_{\mathrm{P}}^{\mathrm{GUD}}(0.809)$ confirms our earlier result that SMSO is a better index to represent the hydrological condition. Figure 12 presents the spatial pattern of the estimated dominant factors for the 17 stations, based on which of the factors (assumed dominant) produced the regression with highest regression coefficient on the GUD in Inner Mongolia. The result shows that generally the water availability is the dominant control (in none of the stations was the temperature found to be dominant) and that the soil-moisture-based index is superior to the precipitation-based index at 12 out of 


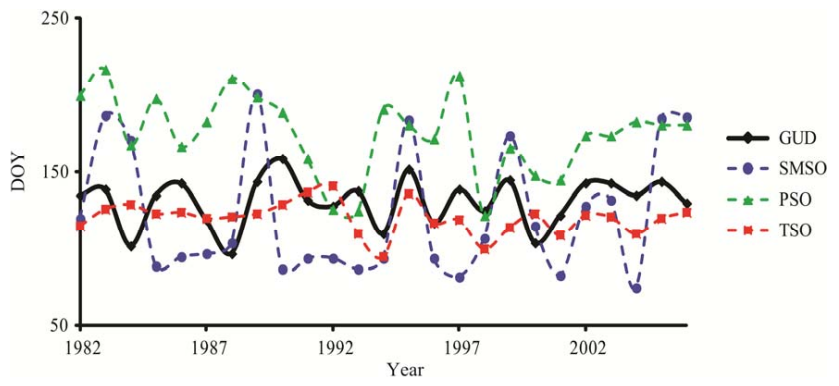

Fig. 9. Time series of SMSO, PSO, TSO, and GUD at station number 10 .

the 17 stations. Even though the water condition is the dominant factor in controlling grass green-up in Inner Mongolia on average, in reality it is indeed possible that the dominant factor may shift inter-annually between water and temperature. Can the same environmental indices considered here be used to predict this inter-annual variability of the alternating controls? This is a question that will be addressed in our future work.

\section{Discussion and conclusions}

Vegetation phenophases, such as spring vegetation green-up, could be influenced by several external factors, of which thermal and water conditions are the two most important ones. The thermal condition is always represented by air temperature, whereas in past remote sensing studies the water condition has been represented by precipitation, not soil moisture, although soil moisture is more directly related to vegetation growth and phenology. To explore the relationships between the environmental factors (e.g., temperature, precipitation) and vegetation green-up, it has been common to accumulate these indicator variables over a given, fixed time span; this is not reasonable physically and contributes to poor predictability. Although one could pick out the dominant factor subjectively retroactively after the fact, there is a lack of an objective way to determine the dominant factors that control phenological behavior.

This paper was motivated by the need to overcome gaps in predictive understanding the environmental controls underpinning vegetation green-up. Soil moisture at a daily time step was estimated over the study region through regional simulations with the distributed VIC model, which provided the means to explore the role of soil moisture directly. Two spring onset dates, SMSO and PSO, were defined in a way similar to the TSO defined by Shen et al. (2011). For each of these three indices, an optimal time span was estimated through a maximizing process as a way of converting the influence of a hydro/thermal condition to an index that estimates a potential GUD, which makes it possible to determine the dominant factor automatically and objectively.

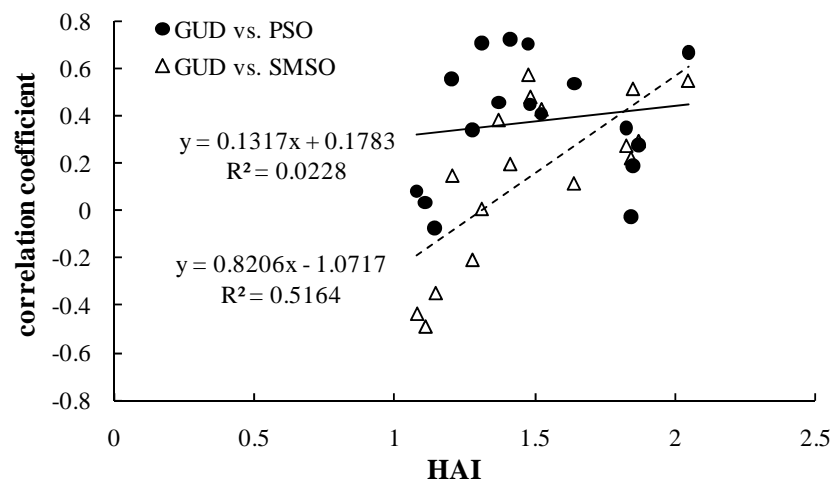

Fig. 10. Trend of correlations between GUD and PSO/SMSO among meteorological stations along a gradient of HAI (the solid circle is the correlation between GUD and PSO, the hollow triangle is the correlation between GUD and SMSO).

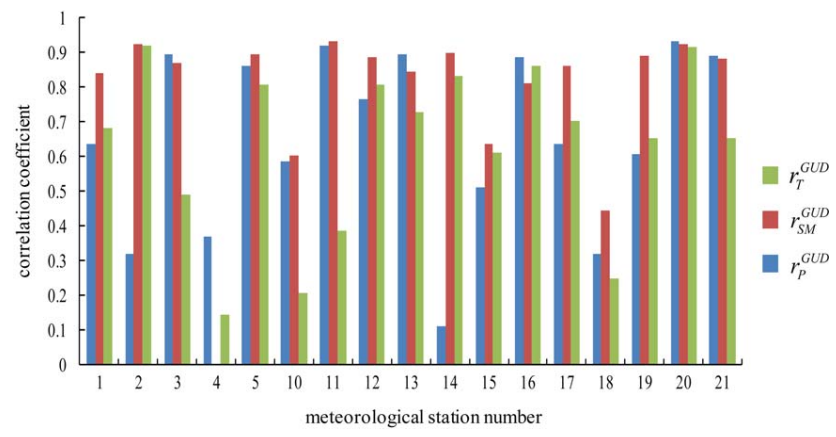

Fig. 11. Correlation coefficients between NDVI-derived GUD and regressed GUDs by assuming different dominant indices among the meteorological stations: green represents the assumption of temperature as dominant control, and red and blue represent that of soil moisture and precipitation, respectively.

We found that the correspondence between the GUD and SMSO showed a significantly better correlation with aridity, as represented by HAI, than that between the GUD and PSO. The cross-correlation between NDVI-derived GUD and GUD values obtained by regression with soil moisture was better than the corresponding value obtained using precipitation data. This showed that the soil-moisturebased index has considerable advantage in regressing grassland spring green-up over the precipitation-based index, and shows good promise for the assessment of potential change in vegetation phenology under climate change. The study also showed that while the dominant environmental factors that govern grass green-up in Inner Mongolia may shift from year to year, on average the water condition appears to be the dominant control over the whole region. The soil moisture used in this study was the output of a hydrological model, whose reliability to predict soil moisture at a specific location at a scale finer than the model grid is open to question, given the potential for considerable sub-grid heterogeneity. Clearly, it cannot be a true substitute for local observations. In spite 


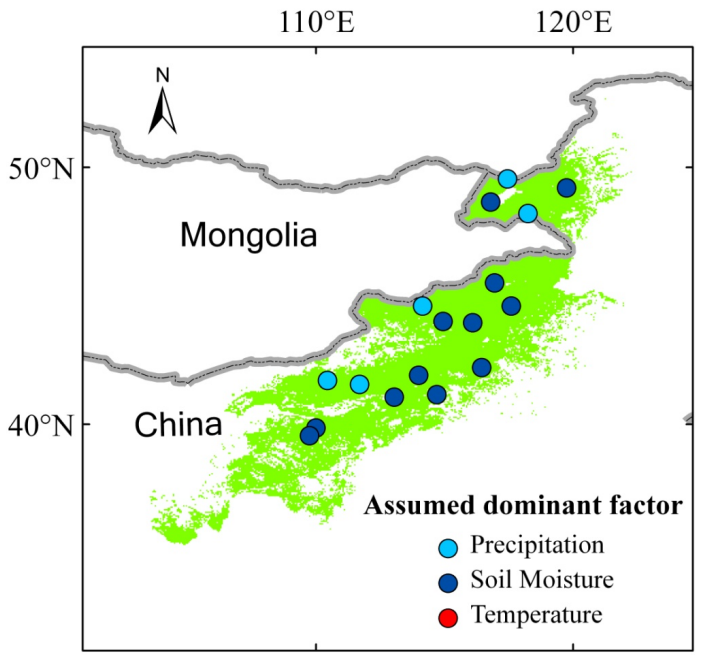

Fig. 12. The spatial pattern of assumed dominant factor based on which could get the best regression of GUD in Inner Mongolia.

of this, the study showed that the soil-moisture-based index was superior to the one based on precipitation. A hydrological model operating at finer space scales would therefore be an improvement, and its pursuit is left for further research. Alternatively, recent advances in remote sensing suggest that they might provide a new method for regional soil moisture monitoring, e.g., microwave remote sensing. However, the depth of penetration of these remote sensing instruments is a concern. For example, the AMSR-E instrument measures soil moisture over the upper $5 \mathrm{~cm}$ of soil (at most) (Rebel et al., 2012), whereas the root zone for grasses over which they extract soil moisture is of the order of $10 \mathrm{~cm}$. These are areas requiring further research.

During the estimation of the various indices (e.g., SMSO) it was found that there was considerable variability in the accumulation time spans between different stations, even though the vegetation type is the same everywhere. This may be due to differences in drainage rates in these locations, which can be caused by differences in topography and soil texture that the vegetation may have acclimatized to. Differences may also be caused by the presence or absence of a water table, exhibiting longer memory, which the vegetation may be able to draw on. These issues require further ecohydrological investigation.

As to the regression/prediction ability, we compared our results with that of the other researchers. The determination coefficient of the regression by Shen is about 0.28 for grassland in Qinghai-Tibetan Plateau (Shen et al., 2011), and that by $\mathrm{Yu}$ et al. (2003) are 0.54 and 0.87 for typical steppe and desert steppe in eastern central Asia. The determination coefficient is 0.78 in our study when assuming soil moisture as the dominant variable. Since the regression regime, variables and accumulation periods are quite different in the studies, the regression ability comparison needs insightful investigation.

Besides soil moisture and temperature that were explored in this study, there could be many other factors that could contribute to the variability of the GUD, such as irradiance, nutrients and oxygen. More detailed investigations at the local level will be needed to explore their roles, relative to the roles of soil moisture and temperature.

Acknowledgements. This research was funded by the National Science Foundation of China (NSFC 51179084, 51190092, 51222901), the special foundation of the Ministry of Water Resources, China (Project No. 201001004), and the foundation of the State Key Laboratory of Hydroscience and Engineering of Tsinghua University (2012-KY-03). Their support is greatly appreciated.

Edited by: S. Thompson

\section{References}

Cayan, D. R., Kemmerdiener, S. A., Dettinger, M. D., Caprio, J. M., and Peterson, D. H.: Changes in the onset of spring in the western United States, B. Am. Meteorol. Soc., 82, 399-415, 2001.

Chen, J., Jönsson, P., Tamura, M., Gu, Z., Matsushita, B., and Eklundh, L.: A simple method for reconstructing a high-quality NDVI time-series data set based on the Savitzky-Golay filter, Remote Sens. Environ., 91, 332-344, 2004.

Czikowsky, M. J. and Fitzjarrald, D. R.: Evidence of seasonal changes in evapotranspiration in eastern U.S. hydrological records, J. Hydrometeorol., 5, 974-988, 2004.

Do, F. C., Goudiaby, V. A., Gimenez, O., Diagne, A. L., Diouf, M., Rocheteau, A., and Akpo, L. E.: Environmental influence on canopy phenology in the dry tropics, Forest Ecol. Manage., 215, 319-328, 2005.

Holdridge, L. R.: Determination of world plant formations from simple climatic data, Science, 105, 367-368, 1947.

Idso, S. B., Jackson, R. D., and Reginato, R. J.: Extending the "degree day" concept of plant phenological development to include water stress effects, Ecology, 59, 431-433, 1978.

Jolly, W. M. and Running, S. W.: Effects of precipitation and soil water potential on drought deciduous phenology in the Kalahari, Global Change Biol., 10, 303-308, 2004.

Julien, Y. and Sobrino, J. A.: Global land surface phenology trends from GIMMS database, Int. J. Remote Sens., 30, 3495-3513, 2009.

Lambers, H., Chapin III, F. S., and Pons, T. L.: Plant Physiology Ecology, 2nd Edn., Springer Science + Business Media, LLC, New York, 2008.

Liang, X., Lettenmaier, D. P., Wood, E. F., and Burges, S. J.: A simple hydrologically based model of land surface water and energy fluxes for general circulation models, J. Geophys. Res., 99, 14415-14428, 1994.

Piao, S., Friedlingstein, P., Ciais, P., Zhou, L., and Chen, A.: Effect of climate and $\mathrm{CO}_{2}$ changes on the greening of the Northern Hemisphere over the past two decades, Geophys. Res. Lett., 33, L23402, doi:10.1029/2006GL028205, 2006. 
Rebel, K. T., de Jeu, R. A. M., Ciais, P., Viovy, N., Piao, S. L., Kiely, G., and Dolman, A. J.: A global analysis of soil moisture derived from satellite observations and a land surface model, Hydrol. Earth Syst. Sci., 16, 833-847, doi:10.5194/hess-16-8332012, 2012.

Reed, B. C., Brown, J. F., VanderZee, D., Loveland, T. R., Merchant, J. W., and Ohlen, D. O.: Measuring phenological variability from satellite imagery, J. Veg. Sci., 5, 703-714, 1994.

Seghieri, J., Vescovo, A., Padel, K., Soubie, R., Arjounin, M., Boulain, N., de Rosnay, P., Galle, S., Gosset, M., Mouctar, A. H., Peugeot, C., and Timouk, F.: Relationships between climate, soil moisture and phenology of the woody cover in two sites located along the West African latitudinal gradient, J. Hydrol., 375, 78-89, 2009.

Sheffield, J. and Wood, E. F.: Characteristics of global and regional drought, 1950-2000: Analysis of soil moisture data from off-line simulation of the terrestrial hydrologic cycle, J. Geophys. Res., 112, D17115, doi:10.1029/2006JD008288, 2007.

Shen, M., Tang, Y., Chen, J., Zhu, X., and Zheng, Y.: Influences of temperature and precipitation before the growing season on spring phenology in grasslands of the central and eastern Qinghai-Tibetan Plateau, Agr. Forest Meteorol., 151, 17111722, 2011.

Shinoda, M., Ito, S., Nachinshonhor, G. U., and Erdenetsetseg, D.: Phenology of Mongolian Grasslands and Moisture Conditions, J. Meteorol. Soc. Jpn., 85, 359-367, doi:10.2151/jmsj.85.359, 2007.

Sparks, T. H., Jeffree, E. P., and Jeffree, C. E.: An examination of the relationship between flowering times and temperature at the national scale using long-term phenological records from the UK, Int. J. Biometeorol., 44, 82-87, 2000.

Thompson, S. E., Harman, C. J., Konings, A. G., Sivapalan, M., Neal, A., and Troch, P. A.: Comparative hydrology across AmeriFlux sites: The variable roles of climate, vegetation, and groundwater, Water Resour. Res., 47, W00J07, doi:10.1029/2010WR009797, 2011.
Wang, J. Y.: A critique of the heat unit approach to plant response studies, Ecology, 41, 785-789, 1960.

Wang, X., Piao, S., Ciais, P., Li, J., Friedlingstein, P., Koven, C., and Chen, A.: Spring temperature change and its implication in the change of vegetation growth in North America from 1982 to 2006, P. Natl. Acad. Sci., 108, 1240-1245, 2011.

Weaver, S. E., Tan, C. S., and Brain, P.: Effect of temperature and soil moisture on time of emergence of tomatoes and four weed species, Can. J. Plant. Sci., 68, 877-886, 1988.

Xie, Z., Yuan, F., Duan, Q., Zheng, J., Liang, M., and Chen, F.: Regional parameter estimation of the VIC land surface model: Methodology and application to river basins in China, J. Hydrometeorol., 8, 447-468, 2007.

Yang, X., Mustard, J. F., Tang, J., and Xu, H.: Regional scale phenology modeling based on meteorological records and remote sensing observations, J. Geophys. Res., 117, G03029, doi:10.1029/2012JG001977, 2012.

Ye, S., Yaeger, M., Coopersmith, E., Cheng, L., and Sivapalan, M.: Exploring the physical controls of regional patterns of flow duration curves - Part 2: Role of seasonality, the regime curve, and associated process controls, Hydrol. Earth Syst. Sci., 16, 44474465, doi:10.5194/hess-16-4447-2012, 2012.

Yu, F., Price, K. P., Ellis, J., and Shi, P.: Response of seasonal vegetation development to climatic variations in eastern central Asia, Remote Sens. Environ., 87, 42-54, 2003.

Yuan, F., Xie, Z., Liu, Q., Yang, H., Su, F., Liang, X., and Ren, L.: An application of the VIC-3L land surface model and remote sensing data in simulating streamflow for the Hanjiang River basin, Can. J. Remote Sens., 30, 680-690, 2004.

Zhang, L. and Liang, Z.: Plant physiology, Science Press, Beijing, 2007.

Zhang, X., Friedl, M. A., Schaaf, C. B., Strahler, A. H., Hodges, J. C. F., Gao, F., Reed, B. C., and Huete, A.,: Monitoring vegetation phenology using MODIS, Remote Sens. Environ., 84, 471-475, 2003. 